\title{
Bees (Hymenoptera: Apoidea) and flowers in natural forest patches of southern Pantanal
}

\author{
Samuel Boff ${ }^{1,3,4}$, Andréa Cardoso Araujo ${ }^{2}$ \& Arnildo Pott $^{2}$ \\ ${ }^{1}$ Programa de Pós-graduação em Biologia Vegetal, Centro de Ciências Biológicas e da Saúde, \\ Universidade Federal de Mato Grosso do Sul - UFMS, Cidade Universitária, \\ CP 549, CEP 79070-900, Campo Grande, MS, Brazil \\ ${ }^{2}$ Centro de Ciências Biológicas e da Saúde, Universidade Federal de Mato Grosso do Sul - UFMS, \\ CP 549, CEP 79070-900, Campo Grande, MS, Brazil. http://www-nt.ufms.br/ \\ ${ }^{3}$ Institute for General Zoology, Martin Luther Universität Halle-Wittenberg, Hoher Weg 8 , \\ D-06120 Halle (Saale), Germany. http://www.uni-halle.del \\ ${ }^{4}$ Corresponding author: Samuel Boff, e-mail: samboff@gmail.com
}

BOFF, S., ARAUJO, A.C. \& POTT, A. Bees (Hymenoptera: Apoidea) and flowers of natural forest patches of southern Pantanal. Biota Neotrop. 13(4): http://www.biotaneotropica.org.br/v13n4/en/ abstract?article+bn00813042013

Abstract: This is the first systematic survey of bees in the Pantanal wetland, Brazil. We recorded bee species and their floral resources within grassland and natural forest edge habitats in the Pantanal. Surveys were conducted during two to four-days per month, for a total of 12 months. Overall, we recorded 56 bee species visiting a total of 63 flowering plants. Asteraceae and Fabaceae presented the highest number of visited species, however Hyptis suaveolens (Lamiaceae) was the plant most visited by different bee species (18). The most generalist native bee was Trigona fuscipennis visiting a total of 17 flowering plant species. Apis mellifera, an exotic bee, visited 27 species. We also report the overlap (37.5\%) in the use of floral resources between the exotic bee Apis mellifera and native bee species. Our updated bee checklist here presented includes 10 new records for the State of Mato Grosso do Sul. These data will support management strategies targeting the conservation of plant-pollinator interactions in the Pantanal ecosystem.

Keywords: bees, floral visitors, forest fragments, surveys

BOFF, S., ARAUJO, A.C. \& POTT, A. Abelhas (Hymenoptera: Apoidea) e flores em fragmentos florestais naturais do Pantanal sul. Biota Neotrop. 13(4): http://www.biotaneotropica.org.br/v13n4/pt/ abstract?article+bn00813042013

Resumo: Esta é a primeira amostragem sistemática de abelhas realizada no Pantanal, Brasil. Nós coletamos espécies de abelhas bem como as plantas que utilizaram como recurso floral em áreas de pastagens naturais e bordas de áreas florestadas. As coletas foram conduzidas durante dois a quatro dias a cada mês, durante 12 meses. Foram registradas 56 espécies de abelhas visitando um total de 63 espécies de plantas. As famílias Asteraceae e Fabaceae apresentaram o maior numero de espécies visitadas, entretanto Hyptis suaveolens (Lamiaceae) foi a espécie visitada pelo maior número de espécies diferentes de abelhas (18). A abelha nativa mais generalista encontrada foi Trigona fuscipennis, que visitou 17 espécies de plantas. Apis mellifera, uma espécie exótica, visitou 27 espécies. Reportamos também a sobreposição (37,5\%) no uso de recursos florais entre A. mellifera e espécies de abelhas nativas. A listagem de abelhas aqui apresentada inclui 10 espécies ainda não mencionadas para o estado de Mato Grosso do Sul. Esses dados apoiarão estratégias de manejo focadas na conservação das interações entre plantas e polinizadores no ecossistema Pantanal.

Palavras-chave: abelhas, visitantes florais, fragmentos florestais, inventários. 


\section{Introduction}

The Brazilian Pantanal, which primarily lies in the state of Mato Grosso do Sul in south-western Brazil, is the largest floodplain on Earth, covering ca. $140000 \mathrm{Km}^{2}$ (Silva \& Abdon 1998) and encompassing a high diversity of habitats (Nunes da Cunha \& Junk 2001). Of the ca. 2000 plant species recorded in this region, few are endemic and the other are typically species with wide geographic distributions (Pott \& Pott 1994, Pott et al. 2011) common to the surrounding ecosystems: Amazonia, Cerrado and Chaco (Prance \& Schaller 1982, Ratter et al. 1988, Pott \& Pott 1994, Nunes da Cunha \& Junk 2001). Furthermore, community dynamics within the Pantanal are dominated by annual hydro-climatic fluctuations (Agência... 2004).

In Brazil, studies on bee fauna have been conducted across a diversity of habitats: the Atlantic Forest (Wilms et al. 1996, Alvesdos-Santos 1999, Ramalho, 2004), the Cerrado (Pedro \& Camargo 1991, Silveira \& Campos 1995, Viana et al. 1997, Andena et al. 2005), the Caatinga (Carvalho \& Marchini 1999, Zanella 2000, Zanella \& Martins 2003, Lorenzon et al. 2003, Aguiar \& Zanella 2005), in sand dunes (Alves-dos-Santos 1999, Viana \& Kleinert 2005), in the southern grasslands (Schlindwein 1998, Alves-dos-Santos 1999, Gonçalves \& Melo 2005, Gonçalves et al. 2009) and in transitional areas of the Cerrado-Amazonia (Santos et al. 2004). Most of these records are concentrated in the eastern part of the country. Therefore, systematics surveys of the bee community including some areas in the Amazon rainforest and the Pantanal wetland are almost unknown (Freitas et al. 2009).

Surveys of species, mainly in areas of little taxonomic knowledge, are essential as baseline data on local biodiversity and species distribution for conservation management (Pinheiro-Machado et al. 2002). Furthermore, bee faunal data or native floral resources for bees are lacking for the State of Mato Grosso do Sul. An exception is a single study (Aoki \& Sigrist 2006), carried out through rapid assessment, which highlights the scarcity of knowledge on the bee fauna in the Mato Grosso do Sul.

In relation to interactions among bees and plants in the Pantanal region, we found only one study, a report on the interactions of the exotic bee Apis mellifera Linnaeus 1758, with plants whose flowers make up its pollen diet (Boff et al. 2011). Despite floristic surveys recording a high richness of melittophilous plants in the Pantanal (Pott \& Pott 1986, Araujo \& Sazima 2003), and preliminary reports of bee visitation for some plant species (Pott \& Pott 1994), no systematic surveys of bee pollinators and their forage plants have been conducted in this region.

Bees play an integral role in maintaining the sexual reproduction and genetic diversity of native and agricultural plant species through pollination services (Kevan \& Imperatriz-Fonseca 2002). The accelerating losses of natural habitats in the State of Mato Grosso do Sul, including the Pantanal, are primarily driven by this region's harboring one of the largest herds of beef cattle in Brazil and thousands kilometers are used mainly for soybean crop (Harris et al. $2005)$. Given the key role bee pollinators play in wild and agricultural ecosystems (Kevan \& Phillips 2001, Kevan \& Imperatriz-Fonseca 2002, Murray et al. 2009), we present a list of plant species visited by bees and species of bees occurring in natural forest patches in the Brazilian Pantanal wetland. This is the first reported survey of bees from Southern Pantanal, representing new records of some species for the state of Mato Grosso do Sul.

\section{Material and Methods}

The study was carried out over 12 months (September 2006 to August 2007), in the Miranda and Abobral sub-regions, Corumbá,
Mato Grosso do Sul, Brazil. This area presents tropical sub-humid climate type Aw (Köppen 1948). Annual rainfall in 2006 was 1162 $\mathrm{mm}$, and in 2007 it was $927 \mathrm{~mm}$ (data from Meteorological station from São Bento ranch). There are two well defined seasons: the rainy season (October to March) and the dry season (April to September), with a mean annual temperature of $26^{\circ} \mathrm{C}$.

Data were gathered in ten natural forest patches at São Bento ranch $\left(19^{\circ} 34^{\prime} 36^{\prime}\right.$ S S, $57^{\circ} 01^{\prime} 08^{\prime}$ 'W). The forest islets are natural patches of forest vegetation presenting circular or elliptic shapes, lying 1 to $3 \mathrm{~m}$ above the surrounding floodable grasslands (Figure 1). These areas remain dry during the high water periods of the Pantanal, and can be viewed as refuges, where plant species intolerant to inundation can grow (Oliveira-Filho \& Martins 1991). Vegetation is primarily composed of riparian forest and Chaco species at the edges, and species typical of semi-deciduous and deciduous forests in the center (Prance \& Schaller 1982). The São Bento ranch is a private propriety where the main activity is cattle ranching. Forest habitats are restricted to these islets and to the riparian forest of the Abobral River, which crosses the ranch.

The studied forest islets are inserted in a matrix of herbaceous/ shrubby species (mainly Sebastiania hispida (Mart.) Pax ex Engl.) that grow in the seasonally flooded grasslands (Figure 2). For most of the year this matrix, which is typically non-managed grassland, serves as a natural pasture for cattle on ranches in the Pantanal. As natural pasture areas extend up to the edges of forest islets, many plants consumed by cattle are less abundant, so favoring occurrence of non-grazed pioneer and ruderal plant species. Nevertheless, some forage species, such as Bidens gardneri Baker (Asteraceae), regrow and flower afterwards, or like Licania parvifolia Huber (Chrysobalanaceae) and Helicteres guazumaefolia (Malvaceae), stay partially out of reach of cows around the forest islets, thus not being consumed by them.

We collected bees and flowers at the edges of the forest islets through two to four - day expeditions per month. Due to access difficulties during the flooding period, when the road access and some areas around of the studied islets remained flooded, some of the chosen forest islets could not be sampled every month. As a result, two forest islets were sampled for 12 months (September/2006August/2007), another two islets for four months (September to December/2006), other two only during three months (September

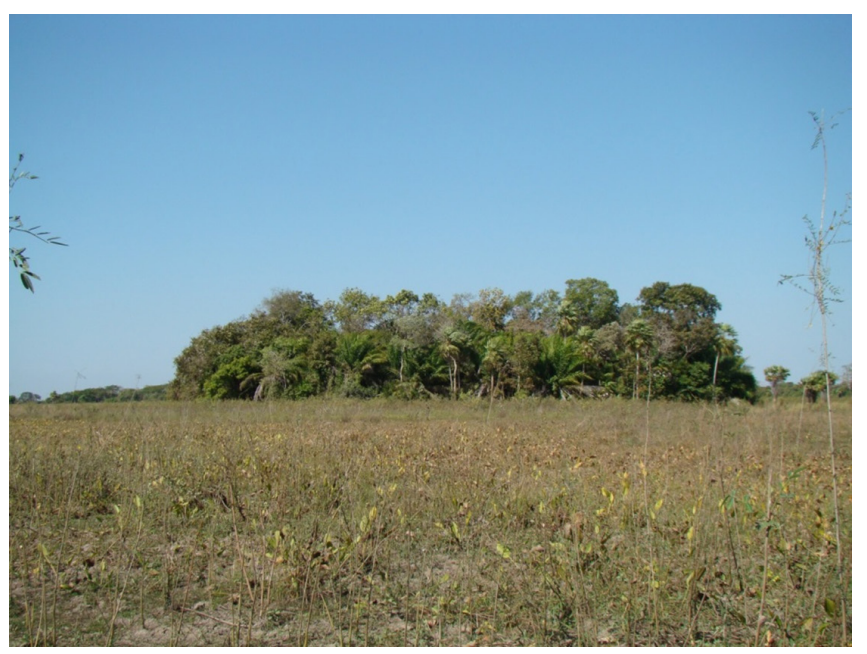

Figure 1. Studied natural patch of forest vegetation (forest islet). Vegetation of fragments is primarily composed of riparian forest and Chaco species at the edges, and species typical of deciduous and semi-deciduous forests in its central portion, São Bento ranch, Pantanal, Brazil. 
to November/2006) and four forest islets for eight months (January to August/2007) (Figure 3).

We established four permanent plots along the edge of each forest islet. All plots were $20 \mathrm{~m}$ long and $5 \mathrm{~m}$ wide $\left(100 \mathrm{~m}^{2}\right)$. The plots were oriented according to the compass points, i.e., one in the North position of the forest islets, one on the East, one on the West and another in the South, adding to $400 \mathrm{~m}^{2}$ sampling area on each forest islet. In each plot, all flowering species whose flowers stayed up to $3 \mathrm{~m}$ high were monthly recorded and assorted by habit.

Focal observations were made between 08:00 $\mathrm{h}$ and 17:00 $\mathrm{h}$. The method used to survey bees in our study differs from the survey sampling described by Sakagami et al. (1967). Here, the bees were qualitatively recorded by one sampler, inside the same plot where plants were monthly monitored regarding to their flowering phenology. Bees were surveyed by focal observation of 10 minutes per flowering individual for each flowering species, inside a given plot or, in case of species occurring in patches, the same amount of time was spent observing flowers in the patch. The collection effort (CE) was calculated based on the formula,

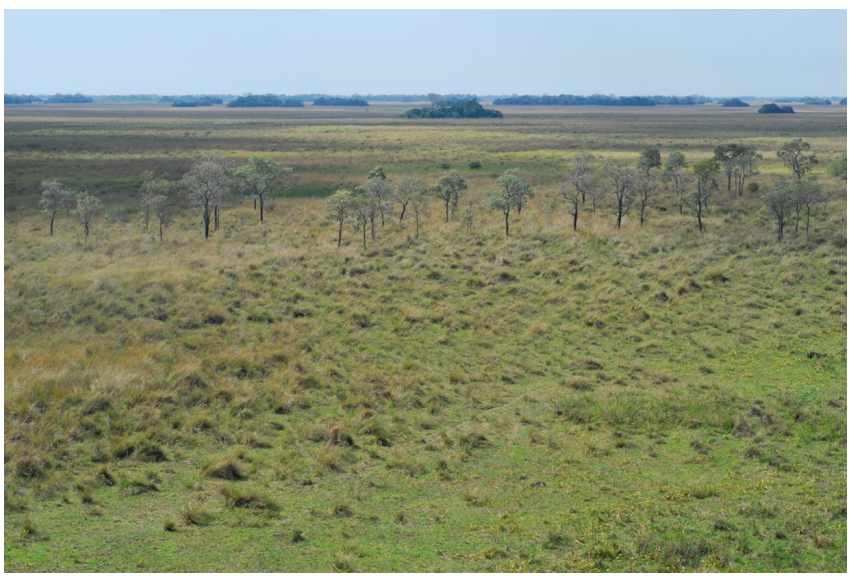

Figure 2. The studied forest islets are inserted in a matrix mainly composed by herbaceous and shrubby species that grow in the seasonally flooded grasslands in the Pantanal, Brazil.
$\left.\mathrm{CE}=\sum n f_{s}\right] \times 10 \div 60$, where the sum of the total number of flowering species (nfs) per plot of a given forest islet, was multiplied by the duration of focal observation $(10 \mathrm{~min})$ and divided by 60 to transform the collection effort into hours. We recorded the identity of the visitor and, when possible the collected resources, plus its role as a possible pollinator. A bee was considered a potential pollinator $(P P)$ if it was observed contacting the flower's reproductive structures during the visit. We used a conservative method to designate a species as $P P$ considering data just when the visitor was collected. After data recording, bees (except Apis mellifera, given its easy identification in the field) were collected with an insect net and put in a killing jar with ethyl acetate.

The collected botanical material was placed in the Herbarium CGMS at the Universidade Federal de Mato Grosso do Sul. The bee specimens were placed in the Laboratory of Zoology, in the same institution and in the personal collection of Dr. Sebastião Laroca who, in collaboration with Dr. Danuncia Urban (both from Universidade Federal do Paraná), identified the bee species. Data referring to the geographic distribution of the bee species in the studied region were compiled from Aoki \& Sigrist (2006) and Moure`s Bee Catalogue, online version (Moure et al. 2008).

\section{Results}

We recorded 187 flowering species, of which 63 species, belonging to 26 plant families, were recorded as being visited by native and exotic bees Apis mellifera. Asteraceae and Fabaceae presented the highest number of visited species, with eight and seven species, respectively, followed by Malvaceae and Rubiaceae (five species each) and Lamiaceae and Verbenaceae (four and three species, respectively). Concerning plant habit, most species visited by bees were herbs $(52 \%)$, followed by trees $(19 \%)$, shrubs $(13 \%)$, liana $(9 \%)$ and sub-shrubs $(6 \%)$. A mean of $22( \pm 5.65)$ visited plant species was recorded per month. The lowest number of blooming plants was recorded in March (10), compared to the highest number recorded in November and December (28 each month). The plants visited by the highest number of bee species were ruderal, such as Hyptis suaveolens (L.) Poit. (18 species), Centrosema brasilianum (L.) Benth. (seven species), Sphagneticola brachycarpa Pruski (six

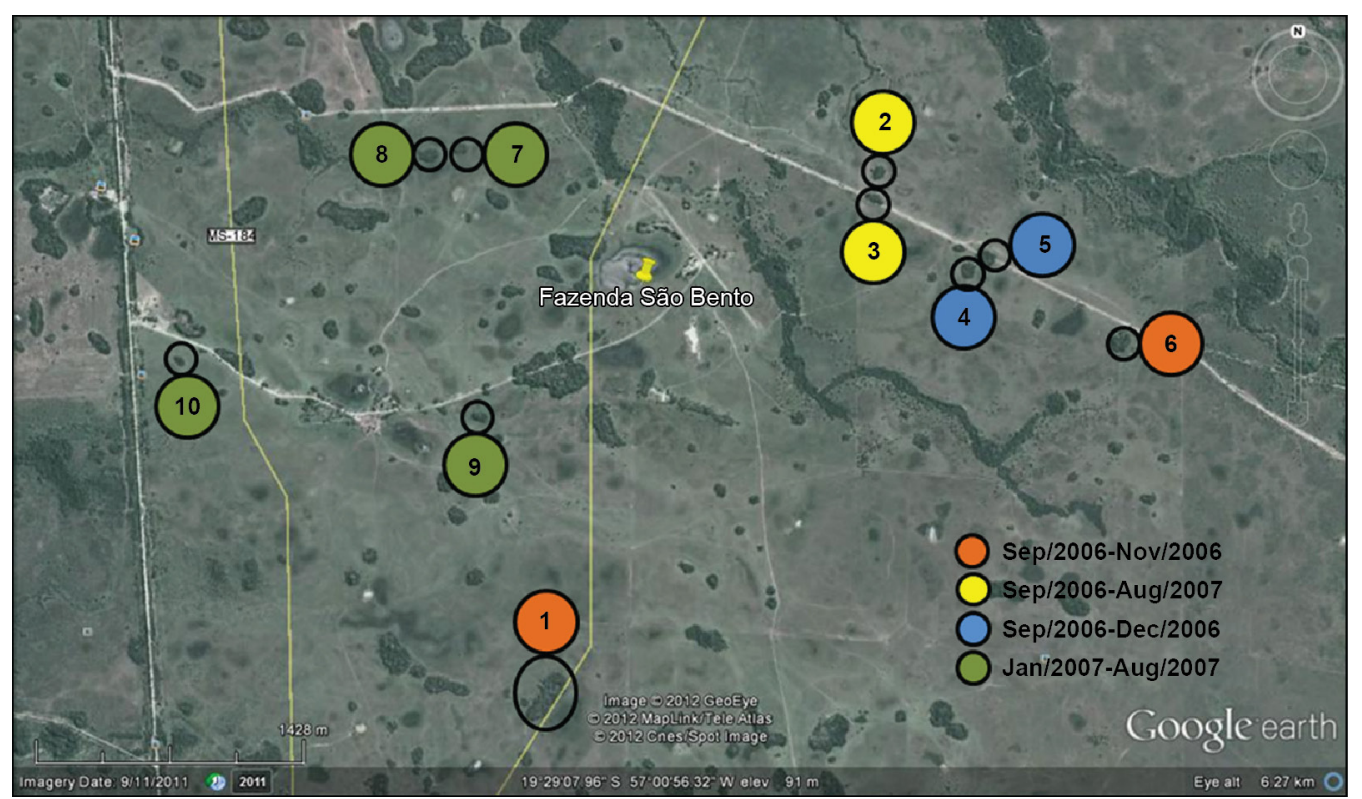

Figure 3. Location of the studied areas at São Bento ranch, southern Pantanal, Brazil. Data collection was conducted between September 2006 and August 2007. The highlighted areas show sampling periods for each islet. Image: modified from Google Earth 2011. 
Table 1. Visited plant species, flowering period observed (F.P.O.), families and species of bees visitor during study in the Pantanal, Brazil. Plants are also classified according to the type of floral resource $(\mathrm{r})$ searched as: nectar $(\mathrm{N})$, pollen $(\mathrm{P})$, oil $(\mathrm{O})$ and mixed $(\mathrm{P}+\mathrm{N}$ or $\mathrm{P}+\mathrm{O})$. Floral visitors are sorted into potential pollinator (po) or plunderer (pl), and when it was not possible to identify its potential role in pollination, no comments were added . NC=Number of bee specimens collected. (*first record of the bee species for Mato Grosso do Sul).

\begin{tabular}{|c|c|c|c|c|c|c|c|c|c|}
\hline Plant family & Species & F.P.O. & Habit & Bee family & Species & $\mathbf{r}$ & po & pl & $\mathrm{NC}$ \\
\hline Amaranthaceae & $\begin{array}{l}\text { Pfaffia glomerata } \\
\text { (Spreng.) Pedersen }\end{array}$ & Jan-July & Herb & Apidae & $\begin{array}{l}\text { Bombus pauloensis } \\
\text { Friese } 1913\end{array}$ & & $\mathrm{X}$ & & 1 \\
\hline Apocynaceae & $\begin{array}{l}\text { Thevetia bicornuta Muell. } \\
\text { Arg. }\end{array}$ & Nov-Feb & Shrub & Apidae & $\begin{array}{l}\text { Ceratina morrensis } \\
\text { Strand, } 1910\end{array}$ & $\mathrm{~N}$ & & & 1 \\
\hline & & & & Apidae & Bombus pauloensis & $\mathrm{N}$ & $\mathrm{X}$ & & 1 \\
\hline Arecaceae & $\begin{array}{l}\text { Attalea phalerata Mart. ex } \\
\text { Spreng. }\end{array}$ & Sept & Tree & Apidae & $\begin{array}{l}\text { Trigona fuscipennis } \\
\text { Friese, } 1900\end{array}$ & $\mathrm{P}$ & $X$ & & 1 \\
\hline \multirow[t]{28}{*}{ Asteraceae } & Bidens gardneri Baker & Feb/May-Aug & Herb & Apidae & Bombus pauloensis & $\mathrm{N}$ & & & 1 \\
\hline & $\begin{array}{l}\text { Centratherum punctatum } \\
\text { Cass. }\end{array}$ & Dec/Apr & Herb & & Apis mellifera & & & & \\
\hline & $\begin{array}{l}\text { Chromolaena odorata (L.) } \\
\text { R.M. King \& H. Rob. }\end{array}$ & $\begin{array}{l}\text { Nov-Feb/May- } \\
\text { June }\end{array}$ & Shrub & Apidae & Bombus pauloensis & $\mathrm{N}$ & & $X$ & 2 \\
\hline & $\begin{array}{l}\text { Conyza bonariensis (L.) } \\
\text { Cronq. }\end{array}$ & Dec-Feb & Herb & Apidae & Apis mellifera & & & & \\
\hline & & & & Apidae & Trigona fuscipennis & $\mathrm{P}$ & & & 6 \\
\hline & & & & Apidae & $\begin{array}{l}\text { Tetragonisca angustula } \\
\text { Latreille, } 1811\end{array}$ & $\mathrm{P}+\mathrm{N}$ & & & 3 \\
\hline & & & & Halictidae & Dialictus sp. & & & & 1 \\
\hline & & & & Halictidae & $\begin{array}{l}\text { *Dialictus ypirangensis } \\
\text { (Schrottky, 1910) }\end{array}$ & & & & 1 \\
\hline & & & & Megachilidae & $\begin{array}{l}\text { Coelioxys vidua Smith. } \\
\text { F (1854) }\end{array}$ & $\mathrm{P}$ & $\mathrm{X}$ & & 1 \\
\hline & $\begin{array}{l}\text { Lepidaploa remotiflora } \\
\text { (Rich.) H. Rob. }\end{array}$ & Feb/Apr & Herb & Apidae & Apis mellifera & & & & \\
\hline & $\begin{array}{l}\text { Porophyllum lanceolatum } \\
\text { DC. }\end{array}$ & May-June & Herb & Apidae & Trigona fuscipennis & & & & 1 \\
\hline & $\begin{array}{l}\text { Praxelis clematidea } \\
\text { R.M. King \& H. Rob. }\end{array}$ & $\begin{array}{l}\text { Sept-Feb/May- } \\
\text { Aug }\end{array}$ & Herb & Halictidae & Augochloropsis sp.1 & & & & 1 \\
\hline & & & & Megachilidae & Megachile sp. 1 & $\mathrm{~N}$ & & & 1 \\
\hline & & & & Apidae & Apis mellifera & & & & \\
\hline & & & & Apidae & Bombus pauloensis & $\mathrm{N}$ & & & 1 \\
\hline & & & & Apidae & Trigona fuscipennis & $\mathrm{P}$ & & & 1 \\
\hline & & & Herb & Apidae & Apis mellifera & & & & \\
\hline & $\begin{array}{l}\text { Sphagneticola } \\
\text { brachycarpa (Baker) } \\
\text { Pruski }\end{array}$ & Sept-Jan & & & & & & & \\
\hline & & & & Apidae & Ceratina sp. 1 & & & & 2 \\
\hline & & & & & & $\mathrm{N}$ & & & \\
\hline & & & & Apidae & $\begin{array}{l}\text { Nannotrigona } \\
\text { testaceicornis } \\
\text { (Lepeletier, 1836) }\end{array}$ & $\mathrm{N}$ & & & 1 \\
\hline & & & & Apidae & Ceratina morrensis & $\mathrm{N}$ & $\mathrm{X}$ & & 1 \\
\hline & & & & Halictidae & Augochlora sp.1 & $\mathrm{N}$ & & & 1 \\
\hline & & & & Megachilidae & $\begin{array}{l}\text { *Coelioxys pampeana } \\
\text { Holmberg, } 1887\end{array}$ & $\mathrm{~N}$ & & & 1 \\
\hline & $\begin{array}{l}\text { Stilpnopappus } \\
\text { pantanalensis } \mathrm{H} . \text { Rob }\end{array}$ & Dec/Apr & Herb & Apidae & Apis mellifera & & & & \\
\hline & $\begin{array}{l}\text { Vernonanthura brasiliana } \\
\text { (L.) H. Rob. }\end{array}$ & Jul-Sept & Herb & Apidae & Apis mellifera & & & & \\
\hline & & & & Apidae & Bombus pauloensis & & & & 1 \\
\hline & & & & Megachilidae & $\begin{array}{l}\text { *Larocanthidium } \\
\text { nigritulum Urban, } 1997\end{array}$ & & & & 1 \\
\hline \multirow[t]{2}{*}{ Bignoniaceae } & $\begin{array}{l}\text { Fridericea florida (DC.) } \\
\text { L.G. Lohmann }\end{array}$ & Oct/ May-Jun & Tree & Apidae & Ceratina morrensis & & & $\mathrm{X}$ & 1 \\
\hline & & & & Apidae & Xylocopa sp. & $\mathrm{N}$ & $\mathrm{X}$ & & 4 \\
\hline Chrysobalanaceae & $\begin{array}{l}\text { Couepia uiti } \\
\text { (Mart. Et Zucc.) Bth. }\end{array}$ & Sept/Nov-Jan & Tree & Apidae & $\begin{array}{l}\text { Paratrigona lineata } \\
\text { Lepeletier, } 1836\end{array}$ & $P$ & & $\mathrm{X}$ & 1 \\
\hline
\end{tabular}


Boff, S. et al.

Table 1. Continued...

\begin{tabular}{|c|c|c|c|c|c|c|c|c|c|}
\hline Plant family & Species & F.P.O. & Habit & Bee family & Species & $\mathbf{r}$ & po & pl & $\mathrm{NC}$ \\
\hline & $\begin{array}{l}\text { Licania parviflora (Sag.) } \\
\text { Fritsch }\end{array}$ & Nov-Jan & Tree & Apidae & Apis mellifera & $\mathrm{N}$ & $\mathrm{X}$ & & \\
\hline & & & & Apidae & Bombus pauloensis & $\mathrm{P}$ & & & 1 \\
\hline & & & & Apidae & Tetragonisca angustula & $\mathrm{P}$ & & & 1 \\
\hline & & & & Apidae & Trigona fuscipennis & & & & 1 \\
\hline Combretaceae & Combretum laxum Jacq. & Jun-Aug & Liana & Apidae & Tetragonisca angustula & $\mathrm{P}$ & & & 1 \\
\hline \multirow[t]{3}{*}{ Commelinaceae } & Commelina erecta $\mathrm{L}$. & Oct-Feb/Apr-Aug & Herb & Apidae & Apis mellifera & & & & \\
\hline & & & & Apidae & Dasyhalonia sp. & $\mathrm{P}$ & $X$ & & 1 \\
\hline & & & & Apidae & Trigona fuscipennis & $P$ & & & 1 \\
\hline \multirow[t]{4}{*}{ Convolvulaceae } & Aniseia cernua Moric. & Mar & Liana & Megachilidae & Pseudocentron sp.2 & $\mathrm{N}$ & & & 1 \\
\hline & $\begin{array}{l}\text { Ipomoea cordatotriloba } \\
\text { Dennst. }\end{array}$ & Sept/Nov & Herb & Apidae & Ceratina morrensis & $\mathrm{N}$ & & & 1 \\
\hline & Ipomoea rubens Choisy & Oct-Feb/Jul-Aug & Liana & Apidae & Ceratina morrensis & $\mathrm{P}+\mathrm{N}$ & & & 1 \\
\hline & Indetermined & & & Megachilidae & Megachile sp. 1 & $\mathrm{~N}$ & & & \\
\hline Erythroxylaceae & $\begin{array}{l}\text { Erythroxylum anguifugum } \\
\text { Mart. }\end{array}$ & Oct/Apr/Aug & Tree & Apidae & Ceratina sp. 1 & & & & 1 \\
\hline & & & & Apidae & Apis mellifera & & & & \\
\hline \multirow[t]{7}{*}{ Euphorbiaceae } & $\begin{array}{l}\text { Croton glandulosus (L.) } \\
\text { M. Arg. }\end{array}$ & Oct-Dec/May-Aug & Herb & Apidae & Apis mellifera & $\mathrm{P}$ & & & \\
\hline & & & & Apidae & $\begin{array}{l}\text { Nannotrigona } \\
\text { testaceicornis }\end{array}$ & $\mathrm{P}$ & & & 1 \\
\hline & & & & Apidae & $\begin{array}{l}\text { Trigona spinipes } \\
\text { Fabricius, } 1793\end{array}$ & $\mathrm{P}$ & & & 1 \\
\hline & & & & Apidae & Trigona fuscipennis & $\mathrm{P}$ & & & 1 \\
\hline & $\begin{array}{l}\text { Sebastiana hispida } \\
\text { (Mart.) Pax ex Engl. }\end{array}$ & Sept-Aug & Herb & Apidae & Trigona fuscipennis & $\mathrm{P}$ & & & 2 \\
\hline & & & & & Bombus pauloensis & $\mathrm{P}$ & & & 1 \\
\hline & & & $\begin{array}{l}\text { Sub- } \\
\text { shrub }\end{array}$ & Apidae & Bombus pauloensis & $\mathrm{P}$ & & & \\
\hline \multirow[t]{19}{*}{ Fabaceae } & & & & Megachilidae & Megachile sp. & $\mathrm{N}$ & $\mathrm{X}$ & & 1 \\
\hline & Aeschynomene histrix Poir. & Sept-Feb & & Apidae & $\begin{array}{l}\text { Exomalopsis analis } \\
\text { Spinola, } 1853\end{array}$ & $\mathrm{~N}$ & $X$ & & 1 \\
\hline & $\begin{array}{l}\text { Canavalia mattogrossensis } \\
\text { (Barb. Rodr.) Malme }\end{array}$ & May-Jun & Liana & Apidae & Trigona fuscipennis & & & & 1 \\
\hline & $\begin{array}{l}\text { Centrosema brasilianum } \\
\text { (L.) Benth. }\end{array}$ & $\begin{array}{l}\text { Sept-Feb/May- } \\
\text { Aug }\end{array}$ & Liana & Apidae & Ceratina morrensis & & & & 2 \\
\hline & & & & Apidae & $\begin{array}{l}\text { *Centris lutea Friese, } \\
1899\end{array}$ & $\mathrm{~N}$ & & & 1 \\
\hline & & & & Apidae & $\begin{array}{l}\text { *Centris xanthocnemis } \\
\text { Perty, } 1833\end{array}$ & $\mathrm{~N}$ & & & 1 \\
\hline & & & & Apidae & Centris sp. 2 & $\mathrm{~N}$ & & & 1 \\
\hline & & & & Apidae & Bombus pauloensis & $\mathrm{N}$ & & & 3 \\
\hline & & & & Apidae & $\begin{array}{l}\text { *Centris aenea } \\
\text { Lepeletier, } 1841\end{array}$ & $\mathrm{~N}$ & & & 1 \\
\hline & & & & Halictidae & $\begin{array}{l}\text { Augochloropsis aff. } \\
\text { cleopatra }\end{array}$ & & & & 1 \\
\hline & Crotalaria micans Link. & Sept/Dec-Jan/July & Herb & Megachilidae & Megachile sp. 1 & $\mathrm{~N}$ & & & 1 \\
\hline & & & & Megachilidae & $\begin{array}{l}\text { Megachile orba } \\
\text { Schrottky, } 1913\end{array}$ & $\mathrm{~N}$ & & & 1 \\
\hline & & & & Apidae & Trigona fuscipennis & $\mathrm{N}$ & & & 1 \\
\hline & & & & Apidae & Xylocopa sp. 1 & $\mathrm{~N}$ & & & 1 \\
\hline & & & & Apidae & $\begin{array}{l}\text { *Xylocopa frontalis } \\
\text { (Olivier, 1789) }\end{array}$ & $\mathrm{N}$ & & & 1 \\
\hline & $\begin{array}{l}\text { Indigofera lespedezioides } \\
\text { Kunth }\end{array}$ & $\begin{array}{l}\text { Sept-Feb/Apr- } \\
\text { June/Aug }\end{array}$ & Herb & Apidae & Centris aenea & $\mathrm{N}$ & & & 1 \\
\hline & Inga vera Kunth & $\begin{array}{l}\text { Sept-Oct/ Apr- } \\
\text { June }\end{array}$ & Tree & Apidae & Trigona fuscipennis & $\mathrm{N}+\mathrm{P}$ & & & 6 \\
\hline & & & & Apidae & Bombus sp. & $\mathrm{N}$ & & & \\
\hline & & & & Apidae & Xylocopa sp. & $\mathrm{N}$ & & & \\
\hline
\end{tabular}


Table 1. Continued...

\begin{tabular}{|c|c|c|c|c|c|c|c|c|c|}
\hline Plant family & Species & F.P.O. & Habit & Bee family & Species & $\mathbf{r}$ & po & pl & NC \\
\hline & $\begin{array}{l}\text { Senna occidentalis (L.) } \\
\text { Link }\end{array}$ & Apr-May & $\begin{array}{l}\text { Sub- } \\
\text { shrub }\end{array}$ & Apidae & Trigona fuscipennis & $P$ & & $\mathrm{X}$ & 1 \\
\hline & & & & Apidae & $\begin{array}{l}\text { Xylocopa ordinaria } \\
\text { Smith, } 1874\end{array}$ & $\mathrm{P}$ & $X$ & & 1 \\
\hline & $\begin{array}{l}\text { Senna splendida (Vogel) } \\
\text { H.S. Irwin \& Barneby }\end{array}$ & Apr-May & Herb & Apidae & Xylocopa frontalis & $P$ & $\mathrm{X}$ & & 2 \\
\hline & & & & Apidae & Trigona fuscipennis & $\mathrm{P}$ & & $\mathrm{X}$ & 2 \\
\hline & & & & Apidae & Xylocopa ordinaria & $\mathrm{P}$ & $X$ & & 1 \\
\hline & & & & & *Centris fuscata & $\mathrm{P}$ & & & 2 \\
\hline & & & Herb & Apidae & Apis mellifera & & & & \\
\hline \multirow[t]{25}{*}{ Lamiaceae } & Hyptis brevipes Baker & $\begin{array}{l}\text { Oct-Nov/Jan-Feb/ } \\
\text { June }\end{array}$ & & Apidae & Centris fuscata & $\mathrm{P}$ & & & $?$ \\
\hline & & & & Halictidae & $\begin{array}{l}\text { Augochloropsis aff. } \\
\text { deianira }\end{array}$ & & & & 1 \\
\hline & $\begin{array}{l}\text { Hyptis suaveolens (L.) } \\
\text { Poit. }\end{array}$ & Sept-Aug & $\begin{array}{l}\text { Sub- } \\
\text { shrub }\end{array}$ & Apidae & Apis mellifera & $\mathrm{N}$ & & & \\
\hline & & & & Megachilidae & Coelioxys pampeana & & & & 1 \\
\hline & & & & Megachilidae & Coelioxys sp. & & & & 1 \\
\hline & & & & Apidae & Ceratina morrensis & & & & 4 \\
\hline & & & & Apidae & Bombus pauloensis & $\mathrm{N}$ & & & 12 \\
\hline & & & & Megachilidae & $\begin{array}{l}\text { Larocanthidium } \\
\text { nigritulum }\end{array}$ & $\mathrm{N}$ & & & 1 \\
\hline & & & & Megachilidae & $\begin{array}{l}\text { Megachile paulistana } \\
\text { Schrottky, } 1902\end{array}$ & $\mathrm{~N}$ & & & 1 \\
\hline & & & & Halictidae & Augochloropsis sp. 3 & & & & 1 \\
\hline & & & & Apidae & $\begin{array}{l}\text { Nannotrigona } \\
\text { testaceicornis }\end{array}$ & $\mathrm{N}+\mathrm{P}$ & & & 1 \\
\hline & & & & Megachilidae & Megachile sp. & $\mathrm{N}$ & & & 1 \\
\hline & & & & Megachilidae & $\begin{array}{l}\text { Coelioxys pirata } \\
\text { Holmberg, E. L. (1884) }\end{array}$ & $\mathrm{N}$ & & & 2 \\
\hline & & & & Halictidae & Agapostemon sp. 1 & & & & 1 \\
\hline & & & & Megachilidae & Coelioxys vidua & $\mathrm{N}$ & & & 1 \\
\hline & & & & Apidae & Ceratinula sp. & & & & 1 \\
\hline & & & & Megachilidae & Coelioxys sp. 2 & $\mathrm{~N}$ & & & 1 \\
\hline & & & & Halictidae & Augochlora sp. 1 & & & & 1 \\
\hline & & & & Megachilidae & Coelioxys sp. 1 & $\mathrm{~N}$ & & & 1 \\
\hline & & & & Apidae & Centris sp. 1 & $\mathrm{~N}$ & & & 1 \\
\hline & & & & Apidae & Tetragonisca angustula & $\mathrm{N}+\mathrm{P}$ & & & 1 \\
\hline & $\begin{array}{l}\text { Marsypianthes } \\
\text { chamaedrys (Vahl) Kuntze }\end{array}$ & Feb/Apr & Herb & Megachilidae & Coelioxys vidua & & & & \\
\hline & $\begin{array}{l}\text { Vitex cymosa Bertero ex } \\
\text { Spreng. }\end{array}$ & Sept-Oct & Tree & Apidae & Apis mellifera & $\mathrm{N}$ & & & \\
\hline & & & & Apidae & *Centris spilopoda & $\mathrm{N}$ & $\mathrm{X}$ & & 1 \\
\hline & & & & Apidae & Trigona fuscipennis & $\mathrm{P}$ & & & 1 \\
\hline \multirow[t]{6}{*}{ Lythraceae } & $\begin{array}{l}\text { Adenaria floribunda } \\
\text { Kunth }\end{array}$ & Oct-Apr & Shrub & Apidae & Apis mellifera & & & & \\
\hline & & & & Halictidae & $\begin{array}{l}\text { Augochloropsis aff. } \\
\text { deianira }\end{array}$ & $\mathrm{P}+\mathrm{N}$ & & & 1 \\
\hline & & & & Colletidae & Hylaeus sp. 1 & & & & 1 \\
\hline & & & & Apidae & Trigona fuscipennis & & & $\mathrm{X}$ & 2 \\
\hline & & & & Apidae & Tetragonisca angustula & $\mathrm{P}$ & & $\mathrm{X}$ & 3 \\
\hline & & & & Apidae & $\begin{array}{l}\text { Nannotrigona } \\
\text { testaceicornis }\end{array}$ & & & & 1 \\
\hline \multirow[t]{5}{*}{ Malpighiaceae } & $\begin{array}{l}\text { Byrsonima orbignyana } \\
\text { A. Juss. }\end{array}$ & Sept-Dec & Tree & Halictidae & Augochloropsis sp. 2 & $P$ & $\mathrm{X}$ & & 1 \\
\hline & & & & Apidae & $\begin{array}{l}\text { Alepidosceles aff. } \\
\text { imitatrix }\end{array}$ & $\mathrm{O}+\mathrm{P}$ & $\mathrm{X}$ & & 1 \\
\hline & & & & Apidae & Tetragonisca angustula & $\mathrm{P}$ & & & 5 \\
\hline & & & & Apidae & Trigona fuscipennis & $\mathrm{P}$ & & & 1 \\
\hline & & & & Apidae & Plebeia sp. 1 & $\mathrm{P}$ & & & \\
\hline
\end{tabular}


Boff, S. et al.

Table 1. Continued...

\begin{tabular}{|c|c|c|c|c|c|c|c|c|c|}
\hline Plant family & Species & F.P.O. & Habit & Bee family & Species & $\mathbf{r}$ & po & pl & $\mathrm{NC}$ \\
\hline \multirow[t]{16}{*}{ Malvaceae } & $\begin{array}{l}\text { Helicteres guazumaefolia } \\
\text { H.B.K. }\end{array}$ & $\begin{array}{l}\text { Sept-Nov/Jan-Apr/ } \\
\text { June/Aug }\end{array}$ & Shrub & Apidae & Trigona fuscipennis & $\mathrm{N}$ & & & 1 \\
\hline & $\begin{array}{l}\text { Melochia graminifolia } \\
\text { A.St. Hil. }\end{array}$ & $\mathrm{Feb} / \mathrm{Apr}$ & Herb & Apidae & Apis mellifera & & & & \\
\hline & Melochia parvifolia Kunth & Oct-Nov/Feb-Aug & Shrub & Apidae & Apis mellifera & $\mathrm{N}$ & & & \\
\hline & & & & Apidae & Bombus pauloensis & & & & 3 \\
\hline & $\begin{array}{l}\text { Melochia simplex A. St. } \\
\text { Hil }\end{array}$ & Annual & Herb & Apidae & Apis mellifera & & & & \\
\hline & & & & Apidae & Bombus pauloensis & $\mathrm{N}$ & $\mathrm{X}$ & & 1 \\
\hline & $\begin{array}{l}\text { Melochia villosa (Mill.) } \\
\text { Fawc. \& Rendle }\end{array}$ & Sept/Nov/Feb-Aug & $\begin{array}{l}\text { Sub- } \\
\text { shrub }\end{array}$ & Apidae & Apis mellifera & & & & \\
\hline & & & & Apidae & Ceratina sp. 1 & $\mathrm{~N}$ & & & 1 \\
\hline & & & & Apidae & $\begin{array}{l}\text { *Ceratina chloris } \\
\text { (Fabricius, 1804) }\end{array}$ & & & & 1 \\
\hline & & & & Apidae & Ceratina morrensis & $\mathrm{N}$ & & & 1 \\
\hline & & & & Apidae & Xylocopa ordinaria & $\mathrm{N}$ & & & 1 \\
\hline & $\begin{array}{l}\text { Sida santaremensis } \\
\text { Monteiro }\end{array}$ & Annual & Herb & Apidae & Apis mellifera & $\mathrm{N}$ & & & \\
\hline & & & & Andrenidae & Anthrenoides sp. 1 & & & & 1 \\
\hline & $\begin{array}{l}\text { Sida viarum } \\
\text { A. St.-Hil. }\end{array}$ & Oct & Herb & Apidae & Apis mellifera & & & & \\
\hline & & & & Apidae & $\begin{array}{l}\text { Centris spilopoda } \\
\text { Moure, } 1969\end{array}$ & $\mathrm{~N}$ & & & 1 \\
\hline & $\begin{array}{l}\text { Ludwigia tomentosa } \\
\text { (Cambess.) H. Hara }\end{array}$ & Dec-Jan/Apr-May & Herb & Apidae & Ceratina morrensis & $\mathrm{N}$ & & & 1 \\
\hline \multirow{3}{*}{ Plantaginaceae } & & Apr & Herb & Halictidae & Dialictus sp. 1 & $\mathrm{P}+\mathrm{N}$ & & & 1 \\
\hline & $\begin{array}{l}\text { Bacopa australis V.C. } \\
\text { Souza }\end{array}$ & & & & & & & & \\
\hline & $\begin{array}{l}\text { Scoparia montevidensis } \\
\text { (Spreng.) R.E.Fr. }\end{array}$ & & Herb & Apidae & Apis mellifera & $\mathrm{P}$ & & & \\
\hline \multirow[t]{3}{*}{ Portulacaceae } & $\begin{array}{l}\text { Portulaca pilosa D. } \\
\text { Legrand }\end{array}$ & $\begin{array}{l}\text { Oct/Jan-Feb/May- } \\
\text { June }\end{array}$ & Herb & Halictidae & Dialictus sp. 1 & & & & 1 \\
\hline & & & & Apidae & Ceratina morrensis & $\mathrm{P}$ & & & 1 \\
\hline & & & & Apidae & $\begin{array}{l}\text { Alepidosceles imitatrix } \\
\text { (Schrottky, 1909) }\end{array}$ & & & & 1 \\
\hline \multirow[t]{12}{*}{ Rubiaceae } & $\begin{array}{l}\text { Borreria eryngioides } \\
\text { Cham. \& Schltdl }\end{array}$ & Out-Dec & Herb & Apidae & Apis mellifera & & & & \\
\hline & $\begin{array}{l}\text { Chomelia pohliana } \\
\text { Müll. Arg. }\end{array}$ & Nov-Dec & Shrub & Apidae & Tetragonisca angustula & $\mathrm{P}$ & & $\mathrm{X}$ & 1 \\
\hline & $\begin{array}{l}\text { Psychotria carthagenensis } \\
\text { Jacq. }\end{array}$ & Out-Feb & Shrub & Megachilidae & Megachile paulistana & & & $\mathrm{X}$ & 1 \\
\hline & $\begin{array}{l}\text { Richardia grandiflora } \\
\text { (Cham. \& Schltdl.) Steud. }\end{array}$ & $\begin{array}{l}\text { Nov-Feb/May- } \\
\text { Aug }\end{array}$ & & Apidae & Apis mellifera & $\mathrm{P}+\mathrm{N}$ & & & \\
\hline & & & Herb & Apidae & Ceratina morrensis & $\mathrm{P}$ & $\mathrm{X}$ & & 1 \\
\hline & & & & Apidae & Bombus pauloensis & $\mathrm{N}$ & & & 1 \\
\hline & & & & Apidae & Ceratina sp. 1 & $\mathrm{~N}+\mathrm{P}$ & & & 1 \\
\hline & $\begin{array}{l}\text { Staëlia thymoides Cham. } \\
\text { et Schltdl. }\end{array}$ & $\begin{array}{c}\text { Sept-Nov/May- } \\
\text { Aug }\end{array}$ & Herb & Apidae & Apis mellifera & & & & \\
\hline & & & & Megachilidae & $\begin{array}{l}\text { Megachile aetheria } \\
\text { Mitchell, } 1930\end{array}$ & $\mathrm{~N}$ & & & 1 \\
\hline & & & & Apidae & Ceratina sp. 1 & $\mathrm{~N}$ & & & 1 \\
\hline & & & & Megachilidae & Coelioxys aff. otomita & $\mathrm{N}$ & & & 1 \\
\hline & $\begin{array}{l}\text { Tocoyena formosa (Cham. } \\
\text { \& Schltdl.) K. Schum. }\end{array}$ & Dec-Jan/Apr & Shrub & Apidae & Trigona fuscipennis & $\mathrm{N}$ & & $\mathrm{X}$ & 2 \\
\hline
\end{tabular}


Table 1. Continued...

\begin{tabular}{|c|c|c|c|c|c|c|c|c|c|}
\hline Plant family & Species & F.P.O. & Habit & Bee family & Species & $\mathbf{r}$ & po & pl & NC \\
\hline Rutaceae & $\begin{array}{l}\text { Zanthoxylum rigidum } \\
\text { Humb. \& Bonpl. ex Willd. }\end{array}$ & Apr/June & Tree & Apidae & Trigona fuscipennis & & & & 1 \\
\hline \multirow[t]{2}{*}{ Salicaceae } & Casearia aculeata Jacq. & Dec/Feb/Apr-May & & Halictidae & Augochloropsis sp. 1 & $\mathrm{P}+\mathrm{N}$ & & & 2 \\
\hline & & & Tree & $\begin{array}{l}\text { Halictidae } \\
\text { Halictidae }\end{array}$ & $\begin{array}{l}\text { Augochloropsis sp. } 2 \\
\text { Augochloropsis sp. } 3\end{array}$ & $\begin{array}{l}\mathrm{P}+\mathrm{N} \\
\mathrm{P}+\mathrm{N}\end{array}$ & & & $\begin{array}{l}2 \\
1\end{array}$ \\
\hline Sapindaceae & $\begin{array}{l}\text { Paullinia pinnata D.R. } \\
\text { Simpson }\end{array}$ & Sept-Oct/Jan/Mar & Liana & Apidae & Ceratina morrensis & $\mathrm{N}$ & & & 1 \\
\hline \multirow[t]{5}{*}{ Solanaceae } & Solanum viarum Dunal & Sept-Apr & Herb & Halictidae & Augochloropsis sp. 1 & $\mathrm{P}$ & $\mathrm{X}$ & & 1 \\
\hline & & & & Apidae & $\begin{array}{l}\text { Centris flavifrons } \\
\text { (Fabricius, 1775) }\end{array}$ & $\mathrm{P}$ & $\mathrm{X}$ & & 1 \\
\hline & & & & & Xylocopa frontalis & $\mathrm{P}$ & $\mathrm{X}$ & & 1 \\
\hline & & & & & Bombus pauloensis & $\mathrm{P}$ & $\mathrm{X}$ & & 4 \\
\hline & & & & Megachilidae & Megachile paulistana & $\mathrm{P}$ & $\mathrm{X}$ & & 1 \\
\hline \multirow[t]{5}{*}{ Verbenaceae } & Lantana canescens Kunth & Dec/Feb & Herb & Apidae & Ceratina sp. 1 & $\mathrm{~N}$ & & & 1 \\
\hline & & & & & Trigona fuscipennis & $\mathrm{P}$ & & & 1 \\
\hline & $\begin{array}{l}\text { Lippia alba (Mill.) N.E.Br. } \\
\text { ex Britton \& P. Wilson }\end{array}$ & Annual & Herb & & Apis mellifera & & & & \\
\hline & $\begin{array}{l}\text { Stachytarpheta } \\
\text { cayennensis(Rich.) Vahl }\end{array}$ & Sept-Feb/July & Herb & Apidae & Apis mellifera & & & & \\
\hline & & & & Apidae & Bombus pauloensis & $\mathrm{N}$ & & & 5 \\
\hline Vochysiaceae & Vochysia divergens Pohl & Aug-Oct & Tree & Apidae & Apis mellifera & & & & \\
\hline
\end{tabular}

species), Crotalaria micans Link and Solanum viarum Dunal (five species each), and pioneer species, such as Adenaria floribunda Kunth and Byrsonima cydoniifolia A. Juss (five species each). The other flowering species were visited by one to four species of bees (Table 1).

We recorded 188 native bees from 55 species representing 27 genera and five families, plus the exotic feral honeybee (Apis mellifera) visiting flowers at the edge of the surveyed forest islets (Table 1) in a total of 306.72 hours of collection effort (Table 2). Native bee species belonged mainly to Apidae (54, 54\%), followed by Megachilidae (ca. 25, 45\%), Halictidae (ca. 16, 36\%), Colletidae and Andrenidae with only $1,81 \%$ each. We recorded the presence of 10 species not yet cited for Mato Grosso do Sul. These species are mainly solitary bees and the most are specialized oil foragers from the tribe Centridini: Centris aenea Lepeletier, 1841, C. flavifrons (Fabricius, 1775), C. fuscata Lepeletier, 1841, C. lutea Friese, 1899, C. spilopoda Moure 1969, C. xanthocnemis Perty 1833, C. sp. 1 and C. sp. 2 (Table 1).

The native bees that visited the greatest diversity of plant species were Trigona fuscipennis Friese 1900 (19 species), Bombus pauloensis Friese 1913 (14 species), Ceratina morrensis Strand 1910 (12 species) and Tetragonisca angustula Latreille, 1811 (seven species). The other bee species visited between one and four flowering species (Table 1).

Amongst the species of plants visited by the native bees, 21 were also visited by Apis mellifera (37.5\% overlapping). The feral honeybee was also an exclusive visitor of seven other species, summing to 27 species, approximately $51 \%$ of the plants that were visited by native bees. Asteraceae and Malvaceae were the families with the highest number of species visited by A. mellifera (seven and five, respectively). Despite the fact that bees were only qualitatively recorded, we noted that $A$. mellifera was in general more common than the other bee species inside the plots, and was recorded throughout the sampling period.

In $25 \%(33 / 132)$ of the recorded interactions, we observed the behavior of the bees on the flowers. In 23 interactions the bees acted as
Table 2. The number of species, specimens and collection effort in the studied areas. $\mathrm{MS}=$ Number of months sampled, $\mathrm{SS}=$ number of specimens sampled, $\mathrm{HCE}=$ Hours of collection effort. $\mathrm{A}=\mathrm{Jan}, \mathrm{B}=\mathrm{Feb}, \mathrm{C}=\mathrm{Mar}, \mathrm{D}=\mathrm{Apr}, \mathrm{E}=\mathrm{May}$, $\mathrm{F}=\mathrm{June}, \mathrm{G}=\mathrm{July}, \mathrm{H}=\mathrm{Aug}, \mathrm{I}=\mathrm{Sept}, \mathrm{J}=\mathrm{Oct}, \mathrm{K}=$ Nov and $\mathrm{L}=$ Dec.

\begin{tabular}{ccccc}
\hline Islet forest & MS & Species & SS & HCE \\
\hline 1 & $3(\mathrm{I}-\mathrm{K})$ & 7 & 16 & 14 \\
2 & $12(\mathrm{I}-\mathrm{H})$ & 17 & 37 & 46.34 \\
3 & $12(\mathrm{I}-\mathrm{H})$ & 16 & 36 & 50 \\
4 & $4(\mathrm{I}-\mathrm{L})$ & 12 & 28 & 21.5 \\
5 & $4(\mathrm{I}-\mathrm{L})$ & 7 & 13 & 23.84 \\
6 & $3(\mathrm{I}-\mathrm{K})$ & 5 & 6 & 13.6 \\
7 & $8(\mathrm{~A}-\mathrm{H})$ & 14 & 17 & 38.34 \\
8 & $8(\mathrm{~A}-\mathrm{H})$ & 11 & 17 & 38.34 \\
9 & $8(\mathrm{~A}-\mathrm{H})$ & 9 & 10 & 31.66 \\
10 & $8(\mathrm{~A}-\mathrm{H})$ & 5 & 6 & 29.10 \\
Total & & & 188 & 306.72 \\
\hline
\end{tabular}

potential pollinators, considering that they contacted the reproductive structures of the flower at the moment of the visit. However, in the 10 remaining interactions the bees just plundered the resource (Inouye 1980). The amounts of each interaction varied among plant/visitor species (Table 1).

\section{Discussion}

In the studied plots, Asteraceae and Fabaceae were the dominant families containing most of the species utilized as a resource by bees; similar to data recorded in Cerrado vegetation (Pedro \& Camargo 1991, Andena et al. 2005). These findings confirm the status of these plant families as important sources of forage for wild bees in these forest islets, as already suggested by the analysis of the pollen diet of Apis mellifera in the same region (Boff et al. 2011). The predominance of herbaceous species in the edge of the forest islets was already 
reported in previous studies (Pott \& Pott 1986, Araujo \& Sazima 2003), but may have been a consequence of only sampling flora up to $3 \mathrm{~m}$ in height. Despite the high number of flowering species in the studied islets $(\mathrm{n}=187)$ and the predominance of melittophilous species (Araujo \& Sazima 2003), the number of plant species visited by bees species, around $35 \%(n=64)$ was lower than reported by previous studies in other Brazilian habitats, such as a transition areas such as the Cerrado-Amazonia in the North (Santos et al. 2004), and in the Southern grasslands (Gonçalves et al. 2009, Pedro \& Camargo 1991, Silveira \& Campos 1995).

Native bees visited less than $30 \%$ of the total of blooming species recorded. However, bee surveying was restricted to the selected plots in this study. Other studies that employed more traditional surveying methodologies, with sampling not restricted to plots, reported a higher number of specimens and species (e.g. Pedro \& Camargo 1991, Alves-dos-Santos 1999, Gonçalves et al. 2009). In a rapid assessment at different habitats in the Cerrado, in Mato Grosso do Sul, Aoki \& Sigrist (2006) reported 113 bee species in 170 hours of collection effort. Although the collection effort (in hours) was less than used in this study, Aoki \& Sigrist (2006) reported twice the number of species recorded here, primarily due to the number of people collecting (eight) in the rapid assessment study. Beyond that in that report surveys were focused solely on the rainy period.

Additionally, the number of open flowers, or floral display, could also affect the abundance and diversity of species observed in this study (Ohashi \& Yahara 2001). Within the studied areas, and excluding some trees species (e.g. Vochysia divergens Pohl, Vitex cymosa Bertero ex Spreng., Licania parviflora) and the herbaceous Hyptis suaveolens and Richardia grandiflora (Cham. \& Schltdl.) Steud., there was a lack of conspicuous floral display, which may have decreased the attractiveness of the forest islets to bees in this area. During surveys in an area with a large floral display of Byrsonima cydoniifolia and Couepia uiti Benth, we observed a lot of individuals from 15 species of Centridini outside the focal plots. Meanwhile, when observing flora of the same species in conditions of lower floral display inside the studied plots, the bees from this tribe were completely absent.

We recorded many ruderal plant species surrounding the forest islets, potentially as a consequence of the annual flooding in the surrounding fields, which eliminates flood-intolerant species. In addition, farmers usually manage the pastures for cattle use and these animals selectively graze the plants at the edges of the forest islets. We observed a high diversity of bee species visiting flowers of ruderal species, e.g. H. suaveolens, in support of previous studies conducted in different habitats (Lorenzon et al. 2003, Fernando César Vieira Zanella, unpublished data). Hyptis suaveolens may potentially be an important floral resource for the studied bee fauna, because it represents a persistent source of pollen and nectar throughout the year for a wide spectrum of bee species (18 in this study). Other abundant ruderal plants recorded were: Borreria eryngioides Cham. \& Schltdl., Conyza bonariensis (L.) Cronquist, Sphagneticola brachycarpa, and mainly Richardia grandiflora; but these species were primarily visited by honeybees.

Bees of the families Apidae, Megachilidae and Halictidae composed, in this order, the highest species richness of bees in this study, similar to reported in other neotropical biogeographical regions such as Cerrado, Caatinga and Amazonia (Silveira \& Campos 1995, Carvalho \& Bego 1996, Viana 1999, Santos et al. 2004.) Studies carried out in Southern Brazil report that species richness of Halictidae is higher than the reported for other areas in Brazil (Barbola \& Laroca 1993, Alves-dos-Santos 1999, Gonçalves et al. 2009), although one study carried out in Rio Grande do Sul reported the Apidae as being the most diverse family (Truylio \& Harter-Marques 2007).
According to the number of visited plant species, the most generalist bees in this study were the species from Apidae, Apis mellifera and Trigona fuscipennis. This result is similar to the observations in areas of Cerrado in São Paulo, where T. spinipes (Fabricius, 1793) and A. mellifera were reported as the most generalist bees (Pedro \& Camargo 1991, Andena et al. 2005). In general, many individuals of these social bees concentrate their forage on several floral resources (Roubik 1989, Kerr et al. 2001). In contrast, nonsocial bees are usually restricted to a lower diversity of plant species (Michener 2007), as recorded in the present study for solitary Apidae like: Centris species, Exomalopsis analis Spinola 1853 and other non-social species from the Halictidae and Megachilidae.

In this study we documented 10 new records of bee species for Mato Grosso do Sul, compared to both Aoki \& Sigrist (2006) and the currently available species list for this region (Moure et al. 2008). This highlights the lack of knowledge about plant-visitors interactions in this state (Pinheiro-Machado et al. 2002, Freitas et al. 2009), and why species with wide distribution in Brazil such as Centris aenea, C. fuscata and C. lutea were not previously recorded in Mato Grosso do Sul. As this is the first systematic survey of the native bee fauna in the Pantanal region, future studies in other habitats, as well as extending surveys to larger areas of forest islets, will certainly add more species to the Pantanal checklist.

The Africanized Apis mellifera was the only floral visitor recorded in seven flowering species and utilized more than $38 \%$ of species visited by native bees. This overlap in flower visitation has been reported in areas of Cerrado in the State São Paulo (Pedro \& Camargo 1991), and to a lesser extent in arid areas in northeastern Brazil (Zanella \& Martins 2003). Plenty of studies show evidences that Apis mellifera affects the reproductive success of different plant species (Gross \& Mackay 1998, Hansen et al. 2002, Carmo et al. 2004) as well as the diversity and abundance of communities of native bees through resource competition (Roubik 1980, 2009, Roubik \& Villanueva-Gutiérrez 2009).

Although this study provides baseline data for bees and their floral resources in the Pantanal wetland, and bee biogeography within Brazil, new studies are still needed for the region. Therefore, given the high conservation value of the Pantanal, in terms of its habitat diversity and of pollinator richness, quantitative surveys of new areas and habitats should be conducted to extend our knowledge on bee species and the pollination services they provide in this region.

\section{Acknowledgements}

We thank the ranch owners for permission access to the study area; Dr. Sebastião Laroca and Dr. Danúnia Urban for identifying the bees; A. Neves, C. Lehn, L. Romero, M. Sazan and W. Guimarães for their assistance in field work. Thank to Dr. Tomás Murray for suggestions on the manuscript and English revision. To CAPES (Coordenação de Aperfeiçoamento de Pessoal de Nível Superior), Fundect (Fundação de Apoio e Desenvolvimento do Ensino, Ciência e Tecnologia do Estado de Mato Gross do Sul) and CNPq (Conselho Nacional de Desenvolvimento Científico e Tecnológico-Programa Ciência sem Fronteiras) provided grants to S. Boff, CNPq and CAPES provided grants to A. Pott; UFMS (Universidade Federal de Mato Grosso do Sul) provided logistical support.

\section{References}

AGUIAR, C.M.L. \& ZANELLA, F.C.V. 2005. Estrutura da comunidade de abelhas (Hymenoptera: Apoidea: Apiformis) de uma área na margem do domínio da Caatinga (Itatim, BA). Neotrop. Entomol. 34(1):15-24. http:// dx.doi.org/10.1590/S1519-566X2005000100003 
ALVES-DOS-SANTOS, I. 1999. Abelhas e plantas melíferas da Mata Atlântica, restinga e dunas do litoral Norte do estado do Rio Grande do Sul, Brasil. Rev. Bras. Entomol. 43 (3-4):191-223.

AGÊNCIA NACIONAL DE ÁGUAS - ANA. 2004. Implementação de práticas de gerenciamento integrado de bacia hidrográfica para o Pantanal e a Bacia do Alto Paraguai: programas de ações estratégicas para o gerenciamento integrado do pantanal e bacia do Alto Paraguai. Athalaia Gráfica e Editora, Brasília, 513p. GEF. Relatório final.

ANDENA, S.R., BEGO, R.S. \& MECHI, M.R. 2005. A comunidade de abelhas (Hymenoptera, Apoidea) de uma área de cerrado (Corumbataí, SP) e suas visitas às flores. Rev. Bras. Zôo. Ciên. 7(1):55-91.

AOKI, C. \& SIGRIST, M.R. 2006. Inventário dos visitantes florais no Complexo Aporé-Sucuriú In Biodiversidade do Complexo AporéSucuriú: subsídios à conservação e manejo do bioma Cerrado (T.C.S Pagotto \& P. R. Souza, eds.). UFMS, Campo Grande, p.143-162. PMid:16404227.

ARAUJO, A.C. \& SAZIMA, M. 2003. The assemblage of flowers visited by hummingbirds in the "capões" of Southern Pantanal, Mato Grosso do Sul, Brazil. Flora 198(6):427-435. http://dx.doi.org/10.1078/0367-2530-00116

BARBOLA, I.F. \& LAROCA, S. 1993. A comunidade de Apoidea (Hymenoptera) da Reserva Passa Dois (Lapa, Paraná, Brasil):Diversidade, abundância relativa e atividade sazonal. Acta Bio. Paranaense. 22(14):91-113.

BOFF, S., LUZ, C.F., ARAÚJO, A.C. \& POTT, A. 2011. Pollen analysis reveals plants foraged by africanized honeybees in the Southern Pantanal, Brazil. Neotrop. Entomol. 40(1):47-54. PMid:21437482. http://dx.doi. org/10.1590/S1519-566X2011000100007

CARMO, R. M., FRANCESCHINELLI, E. V. \& SILVEIRA, F. A. 2004. Introduced honeybees (Apis mellifera) reduce pollination success without affecting the floral resource taken by native pollinators. Biotropica. 36(3):371-376.

CARVALHO, A.M.C. \& BEGO, L.R. 1996. Studies on Apoidea fauna of cerrado vegetation at the Panga Ecological Reserve, Uberlândia, MG, Brazil. Rev. Bras. Entomol. 40(2):147-156.

CARVALHO, C.A.L. \& MARCHINI, L.C. 1999. Plantas visitadas por Apis mellifera L. no vale do rio Paraguaçu, Município de Castro Alves, Bahia. Rev. Bras. Bot. 22(2):333-338. http://dx.doi.org/10.1590/S010084041999000500016

FREITAS, B.M., IMPERATRIZ-FONSECA, V.L., MEDINA, L.M. \& KLEINERT, A.M.P. 2009. Diversity, threats and conservation of native bees in the Neotropics. Apidologie 40(3):332-346. http://dx.doi. org/10.1051/apido/2009012

GONÇALVES, R.B. \& MELO, G.A.R. 2005. A comunidade de abelhas (Hymenoptera, Apidae s. I.) em uma área restrita de campo natural no Parque Estadual de Vila Velha, Paraná: diversidade, fenologia e fontes florais de alimento. Rev. Bras. Entomol. 49(4):557-571. http://dx.doi. org/10.1590/S0085-56262005000400017

GONÇALVES, R.B., MELO, G.A.R. \& AGUIAR, A.J.C. 2009. A assembléia de abelhas (Hymenoptera, Apidae) de uma área restrita de campos naturais do Parque Estadual de Vila Velha, Paraná e comparações com áreas de campos e cerrado. Pap. Avulsos Zool. 49(14):163-181.

GROSS, C.L. \& MACKAY D. 1998. Honeybees reduce fitness in the pioneer shrub Melastoma affine (Melastomataceae). Bio. Conserv. 86(2):169-178 http://dx.doi.org/10.1016/S0006-3207(98)00010-X

HARRIS, M.B., ARCANGELO, C., PINTO, E.C.T., CAMARGO, G., RAMOS NETO, M.B. \& SILVA, S.M. 2005. Estimativas de perda da área natural da Bacia do Alto Paraguai e Pantanal Brasileiro. Conservação Internacional, Campo Grande. Relatório técnico não publicado.

HANSEN, D.M., OLESEN, J.M. \& JONES, C.G. 2002. Trees, birds and bees in Mauritius: exploitative competition between introduced honey bees and endemic nectarivorous birds? J. Biogeogr. 29(5-6):721-734. http:// dx.doi.org/10.1046/j.1365-2699.2002.00720.x

INOUYE, D.W. 1980. The terminology of floral larceny. Ecology 61(5):12511253. http://dx.doi.org/10.2307/1936841
KERR, E.W., CARVALHO, G.A., SILVA, A.C. \& ASSIS, M.G.P. 2001. Aspectos pouco mencionados da biodiversidade Amazônica. Parcerias Estrat. 6(12):21-41.

KEVAN, P.G. \& IMPERATRIZ-FONSECA, V.L., eds. 2002. Pollination bees: the conservation link between agriculture and nature. Ministry of Environment, Brasilia, 313p.

KEVAN, P.G. \& PHILLIPS, T.P. 2001. The economic impacts of pollinator declines: An approach to assessing the consequences. Conserv. Ecol. 5:8. http://www.consecol.org/vol5/iss1/art8/

KÖPPEN, W. 1948. Climatologia. Fondo de Cultura Economica. Buenos Aires. Trad. de Grundriss zu Klimakunde, 1923.

LORENZON, M.C.A., MANTRAGOLO, C.A.R. \& SCHOEREDER, J.H 2003. Flora visitada pelas abelhas Eussociais (Hymenoptera, Apidae) na Serra da Capivara, em Caatinga do Sul do Piauí. Neotrop. Entomol. 32(1):27-36. http://dx.doi.org/10.1590/S1519-566X2003000100004

MICHENER, C.D. 2007. The bees of the world. Baltimore. 2nd ed. The Johns Hopkins University Press, 913p.

MOURE, J.S., MELO, G.A.R. \& DALMOLIN, A. 2008. Catalogue of bees (Hymenoptera, Apoidea) in the Neotropical Region Eletronic. http://www. moure.cria.org.br/catalogue. (último acesso em 03/03/2011).

MURRAY, T.E., KUHLMANN, M. \& POTTS, S.G. 2009. Conservation Ecology of bees: populations, species and communities. Apidologie 40(3):211-236. http://dx.doi.org/10.1051/apido/2009015

NUNES DA CUNHA, C. \& JUNK, W.J. 2001. Distribution of wood plant communities along the flood gradient in the Pantanal of Poconé, Mato Grosso, Brazil. Int. J. Ecol. Environ. Sci. 27(2):63-70.

OHASHI, K. \& YAHARA, T. 2001. Behavioural responses of pollinators to variation in floral display size and their influence on the evolution of floral traits (L. Chittka \& J.D. Thomson, eds.). Cognitive ecology of pollination. Cambridge University Press, p.274-296

OLIVEIRA-FILHO, A.T. \& MARTINS, F.R. 1991. Comparative study of five Cerrado areas in southern Mato Grosso, Brazil. Edinb. J.Bot. 48(3):307332. http://dx.doi.org/10.1017/S0960428600003036

PEDRO, S.R.M. \& CAMARGO, J.M.F. 1991. Interactions on floral resources between the africanized honey bee Apis mellifera $\mathrm{L}$ and the native bee community (Hymenoptera: Apoidea) in a natural "Cerrado" ecosystem in southwest Brazil. Apidologie 22(4):397-415. http://dx.doi.org/10.1051/ apido: 19910405

PINHEIRO-MACHADO, C., ALVES-DOS-SANTOS, I., IMPERATRIZFONSECA, V.L., KLEINERT, A.M.P. \& SILVEIRA, F.A. 2002. Brazilian bee surveys: State of knowledge, conservation and sustainable use. In Pollinating Bees - The conservation link between agriculture and nature (P. Kevan \& V.L. Imperatriz-Fonseca eds.). Ministry of Environment, Brasília, p.115-129

POTT, A. \& POTT, V.J. 1986. Inventário da flora apícola do Pantanal em Mato Grosso do Sul. Embrapa CPAP, Pesquisa em Andamento 3. http:// www.cpap.embrapa.br/publicacoes/online/PA03.pdf.

POTT, A. \& POTT, V.J. 1994. Plantas do Pantanal. Embrapa, Centro de Pesquisa Agropecuária do Pantanal \& Embrapa Serviço de Produção, Brasília, 320p.

POTT, A., OLIVIEIRA, A.K.M., DAMASCENO-JUNIOR, G.A. \& SILVA, J.S.V. 2011. Plant diversity of the Pantanal. Braz. J. Biol. 71(1):265-273. PMid:21537599.

PRANCE, G.T. \& SCHALLER, G.B. 1982. Preliminary study of some vegetation types of the Pantanal, Mato Grosso, Brazil. Brittonia 34(2):228-251. http://dx.doi.org/10.2307/2806383

RAMALHO, M. 2004. Stingless bees and mass flowering trees in the canopy of Atlantic Forest: a tight relationship. Acta Bot. Bras. 18(1):37-47. http:// dx.doi.org/10.1590/S0102-33062004000100005

RATTER, J.A., POTT, A., VALI, J.P., CUNHA, C.N. \& HARIDASAN, M. 1988. Observations on wood vegetation types in the Pantanal and at Corumbá, Brazil. Notes R.B.G. Edinb. 45(3):503-525.

ROUBIK, D.W. 1980. Foraging behavior of competing africanized honeybees and stingless bees. Ecology. 61(4):836-845. http://dx.doi. org/10.2307/1936754 
ROUBIK, D.W. 1989. Ecology and natural history of tropical bees. Cambridge University Press, Cambridge, 514p. http://dx.doi.org/10.1017/ CBO9780511574641

ROUBIK, D.W. \& VILLANUEVA-GUTIÉRREZ, R. 2009. Invasive africanized honey bee impact on native solitary bees: a pollen resource and trap nest analysis. Biol. J. Linn Soc. 98(1):152-160. http://dx.doi. org/10.1111/j.1095-8312.2009.01275.x

ROUBIK, D.W. 2009. Ecological impact on native bees by the invasive africanized honey bee, abejas cleptoparasitas, con énfasis en las abejas hospederas colectoras de aceites (Hymenoptera: Apoidea). Acta Biol. Colomb. 14(2):115-124.

SAKAGAMI S.F., LAROCA, S. \& MOURE, J.S. 1967. Wild bees biocenotics in São José dos Pinhais (PR), South Brazil: Preliminary Report. Jour. Fac. Sci. Hokkaido Uni., Ser. VI, Zool. 16(2):253-91.

SANTOS, F.V., CARVALHO, C.A.L. \& SILVA, R.F. 2004. Diversidade de abelhas em uma área de transição Cerrado-Amazônia. Acta Amazôn. 34(2):319-328. http://dx.doi.org/10.1590/S0044-59672004000200018

SCHLINDWEIN, C. 1998. Frequent oligolecty characterizing a diverse bee-plant community in a xerophytic bushland of subtropical Brazil. Stud. Neotrop. Fauna Environ. 33(1):46-59. http://dx.doi.org/10.1076/ snfe.33.1.46.2168

SILVA, J.S.V. \& ABDON, M.M. 1998. Delimitação do Pantanal brasileiro e suas sub-regiões. Pesqui. Agropecu. Bras. 33(Número especial):1703-1711.

SILVEIRA, F.A. \& CAMPOS, M.J.O. 1995. A melissofauna de Corumbataí (SP) e Paraopeba (MG):uma análise da biogeografia das abelhas do cerrado brasileiro (Hymenoptera: Apoidea). Rev. Bras. Entomol. 39(2):371-401.
TRUYLIO, B. \& HARTER-MARQUES, B. 2007. A comunidade de abelhas (Hymenoptera, Apoidea) em áreas florestais do Parque Estadual de Itapuã (Viamão, RS):diversidade, abundância relativa e atividade sazonal. Iheringea, Séries Zoologia 97(4):392-399. http://dx.doi.org/10.1590/ S0073-47212007000400006

VIANA, B.F., KLEINERT, A.M.P. \& IMPERATRIZ-FONSECA, V.L. 1997. Abundance and flower visits of bees in a Cerrado of Bahia, Tropical Brazil. Stud. Neotrop. Fauna Environ. 32(4):212-219.

VIANA, B.F. 1999. A comunidade de abelhas (Hymenoptera: Apoidea) das dunas interiores do Rio São Francisco, Bahia, Brasil. Neotrop. Entomol. 28(4):635-645.

VIANA, B.F. \& KLEINERT, A.M.P. 2005. A community of flower-visiting bees (Hymenoptera: Apoidea) in the coastal sand dunes of northeastern Brasil. Biota Neotrop. http://www.biotaneotropica.org.br/v5n2/pt/ fullpaper?bn00305022005+en

ZANELLA, F.C.V. 2000. The bees of the Caatinga (Hymenoptera, Apoidea, Apiformes):A species list and comparative notes regarding their distribution. Apidologie 31(5):579-592. http://dx.doi.org/10.1051/ apido: 2000148

ZANELLA, F.C.V. \& MARTINS, C.F. 2003. Abelhas da Caatinga: Biogeografia, ecologia e conservação In Ecologia e Conservação da Caatinga (I.R. Leal, Tabarelli, R. M. \& Silva, J.M.C. eds.). Editora Universitária da UFPE, Recife, p.75-134 .

WILMS, W., IMPERATRIZ-FONSECA, V.L. \& ENGELS W. 1996. Resource partitioning between highly eusocial bees and possible impact of the introduced africanized honey bee on native stingless bees in the Brazilian Atlantic Rainforest. Stud. Neotrop. Fauna Environ. 31(3-4):37-51. http:// dx.doi.org/10.1076/snfe.31.3.137.13336 